\title{
Drug Susceptibility Testing of 31 Antimicrobial Agents on Rapidly Growing Mycobacteria Isolates from China
}

\author{
Hui Pang, ${ }^{1,2,3}$ Guilian Li, ${ }^{3,4}$ Xiuqin Zhao, ${ }^{3,4}$ Haican Liu, ${ }^{3,4}$ Kanglin Wan, ${ }^{3,4}$ and Ping Yu \\ ${ }^{1}$ Department of Immunology, Xiangya School of Medicine, Central South University, Changsha, Hunan 410078, China \\ ${ }^{2}$ Department of Immunology, Changzhi Medical College, Changzhi, Shanxi 046000, China \\ ${ }^{3}$ State Key Laboratory for Infectious Disease Prevention and Control, National Institute for Communicable Disease \\ Control and Prevention, Chinese Center for Disease Control and Prevention, Beijing 102206, China \\ ${ }^{4}$ Collaborative Innovation Center for Diagnosis and Treatment of Infectious Diseases, Hangzhou, Zhejiang 310000, China
}

Correspondence should be addressed to Kanglin Wan; panghuimail@sina.com and Ping Yu; 1084900579@qq.com

Received 29 April 2015; Revised 29 June 2015; Accepted 5 July 2015

Academic Editor: Abdelwahab Omri

Copyright (c) 2015 Hui Pang et al. This is an open access article distributed under the Creative Commons Attribution License, which permits unrestricted use, distribution, and reproduction in any medium, provided the original work is properly cited.

Objectives. Several species of rapidly growing mycobacteria (RGM) are now recognized as human pathogens. However, limited data on effective drug treatments against these organisms exists. Here, we describe the species distribution and drug susceptibility profiles of RGM clinical isolates collected from four southern Chinese provinces from January 2005 to December 2012. Methods. Clinical isolates (73) were subjected to in vitro testing with 31 antimicrobial agents using the cation-adjusted Mueller-Hinton broth microdilution method. The isolates included $55 \mathrm{M}$. abscessus, $11 \mathrm{M}$. fortuitum, $3 \mathrm{M}$. chelonae, $2 \mathrm{M}$. neoaurum, and $2 \mathrm{M}$. septicum isolates. Results. M. abscessus (75.34\%) and M. fortuitum (15.07\%), the most common species, exhibited greater antibiotic resistance than the other three species. The isolates had low resistance to amikacin, linezolid, and tigecycline, and high resistance to first-line antituberculous agents, amoxicillin-clavulanic acid, rifapentine, dapsone, thioacetazone, and pasiniazid. M. abscessus and M. fortuitum were highly resistant to ofloxacin and rifabutin, respectively. The isolates showed moderate resistance to the other antimicrobial agents. Conclusions. Our results suggest that tigecycline, linezolid, clofazimine, and cefmetazole are appropriate choices for M. abscessus infections. Capreomycin, sulfamethoxazole, tigecycline, clofazimine, and cefmetazole are potentially good choices for M. fortuitum infections. Our drug susceptibility data should be useful to clinicians.

\section{Introduction}

Nontuberculous mycobacteria (NTM) form a large class within the Mycobacteriaceae family. More than 100 NTM species are found in soil, potable water, food, and animals [1]. In China, the proportion of NTM among all mycobacterial isolates has increased from $11.1 \%$ to $22.9 \%$ according to National surveys conducted in 1990 and 2010 [2]. Thus, the rising percentage of NTM in China is now an important public health concern $[1,2]$.

NTM can be classified into rapidly growing mycobacteria (RGM) and slowly growing mycobacteria (SGM). More than 50 RGM species are able to produce mature colonies on agar plates within 7 days [3]. Many of these are important human pathogens that cause pulmonary and soft tissue infections and various other infections $[4,5]$. RGM comprise a diverse group of species, including M. abscessus, M. fortuitum, $M$. chelonae, and various rare species. Most studies have shown that $M$. abscessus accounts for $80 \%$ of the lung disease caused by RGM, and after M. fortuitum, $M$ abscessus is the second most common RGM to cause extrapulmonary disease $[3,6]$.

Diagnosing and treating RGM diseases is challenging for clinicians $[6,7]$. Over the past few decades, RGM infections have been diagnosed based on a patient's clinical characteristics, risk factors, and the results of antimicrobial susceptibility testing [3]. However, drug susceptibility patterns vary greatly between RGM species and optimally therapeutic regimens have not been established [3, 6].

In this study, the antimicrobial susceptibility of 73 clinical RGM isolates and their corresponding standard strains were tested with 31 antibiotics. The tests were based on the recommendations of the Clinical and Laboratory Standards 
Institute (CLSI) [8] for determining the clinical criteria for therapeutic treatment of RGM infections.

\section{Materials and Methods}

2.1. Clinical Isolates and Reference Strains. During the period from January 2005 to December 2012, RGM clinical strains were isolated from the sputum specimens of suspected tuberculosis patients in Fujian, Hunan, Gansu, and Sichuan Provinces of China. Eighteen clinical strains were isolated in 2005, 6 in 2006, 8 in 2009, 25 in 2010, 8 in 2011, and 8 in 2012. International-standard RGM species were used as the corresponding quality control strains for the clinical isolates tested herein (i.e., M. abscessus ATCC19977, M. chelonae ATCC35752, M. fortuitum DSM44220, M. neoaurum ATCC25795, and M. septicum DSM44393).

2.2. Species Identification. Species identification of the isolates was conducted by sequence analysis of the $h s p 65$ gene. When an $h s p 65$ sequence match was less than $97 \%$, the $r p o B$ gene and the $16 \mathrm{~S}-23 \mathrm{~S}$ internal transcribed spacer region were also sequenced $[9,10]$. PCR products were sequenced by the Beijing Tsingke Bio Tech Co. Ltd. (Beijing, China). The sequences obtained were compared with those in the GenBank (National Center for Biotechnology Information: http:// www.ncbi.nlm.nih.gov/) DNA sequence database; species identification was confirmed if a $97 \%$ match was achieved $[9,10]$.

2.3. RGM Growth Medium. The strains tested were cultured using Difco Middlebrook 7H10 Agar (BD company). The medium was prepared as follows. First, $19 \mathrm{~g}$ of $7 \mathrm{H} 10$ (powder) was suspended in $900 \mathrm{~mL}$ of purified water containing $5 \mathrm{~mL}$ of glycerol and then mixed thoroughly. Next, the powder was completely dissolved by heating with frequent agitation for $1 \mathrm{~min}$ and then sterilized at $121^{\circ} \mathrm{C}$ for $10 \mathrm{~min}$. Last, $100 \mathrm{~mL}$ of Middlebrook OADC enrichment solution (BD, Franklin Lakes, NJ, USA) was added aseptically to the medium after cooling to $50-55^{\circ} \mathrm{C}$.

2.4. Medium for Drug Susceptibility Testing of RGM. The medium used for antimicrobial susceptibility testing of RGM was BBL cation-adjusted Mueller-Hinton (CAMH) Broth (BD, Franklin Lakes, NJ, USA). The medium was prepared by suspending $22 \mathrm{~g}$ of powder in $1 \mathrm{~L}$ of purified water and autoclaving the bottle at $121^{\circ} \mathrm{C}$ for $10 \mathrm{~min}$, followed by supplementation with $20-25 \mathrm{mg} / \mathrm{L}$ of calcium and $10-12.5 \mathrm{mg} / \mathrm{L}$ of magnesium.

2.5. Drug Susceptibility Tests. The strains were grown on $7 \mathrm{H} 10$ agar and incubated at $37^{\circ} \mathrm{C}$ in ambient air. Drug susceptibility tests were performed using the broth microdilution method according to CLSI recommendations [6]. Tests on the strains were repeated at least twice using 96-well microplates. The final minimum inhibitory concentration (MIC) of each drug used for each strain was the average value of the two tests. Bacterial inocula were adjusted with normal saline to a density of a $0.5 \mathrm{McF}$ arland standard with an organism density of approximately $1 \times 10^{7}$ colony forming units $(\mathrm{CFU}) / \mathrm{mL}$.
Fifty microliters of the suspension, added to $10 \mathrm{~mL}$ of $\mathrm{CAMH}$ broth, was vortexed thoroughly to make a 1:200 bacterial dilution. First, $100 \mu \mathrm{L}$ of CAMH medium was added to each well of a 96-well microplate, except for the first well of the each row. CAMH medium $(180 \mu \mathrm{L})$ was added to the first well of every row, followed by a $20 \mu \mathrm{L}$ aliquot of a drug solution. The thoroughly mixed solution in the first well was serially diluted into the next well, and so on up to the 11th well. The 12th well in every row was a blank control. Second, $100 \mu \mathrm{L}$ of the bacterial dilution was added to the wells of the 96-well microplate. The final volume in each row was $200 \mu \mathrm{L}$. Finally, the 96-well microplate was sealed in a plastic bag and incubated at $37^{\circ} \mathrm{C}$. The concentration ranges for rifampicin, isoniazid, ethambutol, streptomycin, tobramycin, sulfamethoxazole, dapsone, amoxicillinclavulanic acid, cefoxitin, cefmetazole, thioacetazone, pasiniazid, minocycline, doxycycline, tigecycline, and meropenem were all $0.25-256 \mu \mathrm{g} / \mathrm{mL}$, those of amikacin, kanamycin, capreomycin, ofloxacin, ciprofloxacin, levofloxacin, moxifloxacin, sparfloxacin, clarithromycin, azithromycin, roxithromycin, clofazimine, rifapentine, and rifabutin were $0.03-32 \mu \mathrm{g} / \mathrm{mL}$, and the concentration of linezolid was $0.06-$ $64 \mu \mathrm{g} / \mathrm{mL}$. All the drugs were purchased from Sigma-Aldrich (St. Louis, MO). The following two negative controls were used: CAMH broth plus inoculum (drug-free control), which was used to decide the optimal time to add Alamar blue to the assay; the other was only CAMH broth, which was used to decide the interference level of CAMH to Alamar blue. The plates were checked after $72 \mathrm{~h}$. If the drug-free growth control showed sufficient bacterial growth, the indicator ( $20 \mu \mathrm{L}$ of Alamar Blue and $50 \mu \mathrm{L}$ of sterile $5 \%$ Tween-80) turned pink. Generally, the minimal inhibitory concentration (MIC) value was read on day 3 or 4 after addition of the inoculum. If bacterial growth in the drug-free control was insufficient on day 5 , the test was repeated. The MIC values for clarithromycin were evaluated 3 to 5 days after inoculation and were incubated for a further 14 days at $37^{\circ} \mathrm{C}$ for the final reading.

MIC values were defined as the lowest concentration of drug that inhibited the visible growth of the isolates tested. $\mathrm{MIC}_{50}$ and $\mathrm{MIC}_{90}$ values were defined as the drug concentrations at which $50 \%$ and $90 \%$ of the isolates tested showed no visible growth, respectively. The MIC breakpoints of antibiotics displaying susceptibility, intermediate susceptibility, and resistance were interpreted by the World Health Organization (WHO) [11] and CLSI guidelines [8], except for sparfloxacin [12], clofazimine [13], azithromycin [14], roxithromycin [14], amoxicillin-clavulanic acid [15], cefmetazole [16], rifapentine [17], rifabutin [17], dapsone [18], and thioacetazone [19] (Table 1).

2.6. Statistical Analysis. The data were analyzed by SPSS17.0 software. The drug susceptibility percentages and the $\mathrm{MIC}_{50}$ and $\mathrm{MIC}_{90}$ among the antimicrobial agents tested were determined for the five species of RGM isolates ( $M$. abscessus, M. chelonae, M. fortuitum, M. neoaurum, and M. septicum). 
TABLE 1: MIC $(\mu \mathrm{g} / \mathrm{mL})$ breakpoints of 31 antimicrobial agents.

\begin{tabular}{|c|c|c|c|}
\hline \multirow[b]{2}{*}{ Antibacterial agents } & \multicolumn{3}{|c|}{ MIC breakpoints } \\
\hline & Susceptibility & $\begin{array}{l}\text { Intermediate } \\
\text { susceptibility }\end{array}$ & Resistance \\
\hline Rifampicin & - & - & $\geqslant 1[8]$ \\
\hline Isoniazid & - & - & $\geqslant 1[8]$ \\
\hline Ethambutol & - & - & $\geqslant 4[8]$ \\
\hline Streptomycin & - & - & $\geqslant 5[8]$ \\
\hline Amikacin & $\leqslant 16$ & 32 & $\geqslant 64[8]$ \\
\hline Kanamycin & - & - & $\geqslant 4[11]$ \\
\hline Capreomycin & - & - & $\geqslant 2.5[11]$ \\
\hline Tobramycin & $\leqslant 2$ & 4 & $\geqslant 8[8]$ \\
\hline Ofloxacin & - & - & $\geqslant 2[11]$ \\
\hline Ciprofloxacin & $\leqslant 1$ & 2 & $\geqslant 4[8]$ \\
\hline Levofloxacin & $\leqslant 2$ & 4 & $\geqslant 8[8]$ \\
\hline Sparfloxacin & $\leqslant 1$ & 2 & $\geqslant 4[12]$ \\
\hline Moxifloxacin & $\leqslant 1$ & 2 & $\geqslant 4[8]$ \\
\hline Linezolid & $\leqslant 8$ & 16 & $\geqslant 32[8]$ \\
\hline Clofazimine & - & - & $\geqslant 1[13]$ \\
\hline Sulfamethoxazole & $\leqslant 38$ & - & $\geqslant 76[8]$ \\
\hline Minocycline & $\leqslant 1$ & $2-4$ & $\geqslant 8[8]$ \\
\hline Doxycycline & $\leqslant 1$ & $2-4$ & $\geqslant 8[8]$ \\
\hline Tigecycline & $\leqslant 1$ & $2-4$ & $\geqslant 8[8]$ \\
\hline Clarithromycin & $\leqslant 2$ & 4 & $\geqslant 8[8]$ \\
\hline Azithromycin & $\leqslant 2$ & 4 & $\geqslant 8[14]$ \\
\hline Roxithromycin & $\leqslant 2$ & 4 & $\geqslant 8[14]$ \\
\hline $\begin{array}{l}\text { Amoxicillin- } \\
\text { clavulanic } \\
\text { acid }\end{array}$ & $\leqslant 8 / 4$ & $16 / 8$ & $\geqslant 32 / 16[15]$ \\
\hline Cefoxitin & $\leqslant 16$ & $32-64$ & $\geqslant 128[8]$ \\
\hline Cefmetazole & $\leqslant 16$ & 32 & $\geqslant 64[16]$ \\
\hline Meropenem & $\leqslant 4$ & $8-16$ & $\geqslant 32[8]$ \\
\hline Rifapentine & - & - & $\geqslant 1[17]$ \\
\hline Rifabutin & - & - & $\geqslant 1[17]$ \\
\hline Dapsone & - & - & $\geqslant 4[18]$ \\
\hline Thioacetazone & - & - & $\geqslant 8[19]$ \\
\hline Pasiniazid & - & - & $\geqslant 2[11]$ \\
\hline
\end{tabular}

\section{Results}

Among the 73 clinical isolates, 55 (75.34\%) were M. abscessus, 11 (15.07\%) were $M$. fortuitum, 3 (4.11\%) were $M$. chelonae, 2 (2.74\%) were $M$. neoaurum, and 2 (2.74\%) were M. septicum. Of the isolates, 63 were from Fujian, 7 were from Hunan, 2 were from Gansu, and 1 was from Sichuan.

The antimicrobial susceptibility profiles of the five reference RGM strains are shown in Table 2. The strains were highly resistant to the four first-line antituberculous agents tested on them, especially M. chelonae, M. abscessus, and $M$. fortuitum. We found that aminoglycoside antibiotics including amikacin, kanamycin, capreomycin, and tobramycin, were effective antimicrobials for the RGM species. However, M. abscessus was resistant to tobramycin and $M$. chelonae was resistant to kanamycin and capreomycin. Fluoroquinolones (including ofloxacin, ciprofloxacin, levofloxacin, sparfloxacin, and moxifloxacin) also exhibited favorable in vitro activities against the standard RGM strains. However, $M$. chelonae was resistant to all four of the fluoroquinolones we tested. M. chelonae and M. abscessus were not susceptible to minocycline and doxycycline, but were susceptible to tigecycline. Clarithromycin, azithromycin, and roxithromycin all exhibited favorable in vitro activities (except for azithromycin) against $M$. septicum. The reference species were not susceptible to amoxicillin-clavulanic acid but were susceptible or moderately susceptible to cefoxitin and cefmetazole, apart from $M$. chelonae and M. septicum, which were not susceptible to cefoxitin. Unlike $M$. chelonae, the RGM species were susceptible to meropenem. Rifapentine had a more favorable MIC than rifabutin against $M$. chelonae, M. abscessus, and M. fortuitum. Linezolid, clofazimine, and sulfamethoxazole were highly active against the standard RGM organisms. However, dapsone, thioacetazone, and pasiniazid displayed poor activities against the reference species.

The percentage of in vitro drug susceptibility values of the 31 antibacterial agents against the 73 clinical RGM isolates is shown in Table 3. Among the four first-line antituberculous drugs, no $(0 / 73)$ strains were susceptible to isoniazid and 3 $(4.11 \%), 2(2.74 \%)$, and $4(5.48 \%)$ strains were susceptible to rifampicin, ethambutol, and streptomycin, respectively. However, the $M$. chelonae isolates were less resistant to rifampicin than M. abscessus and M. fortuitum. Aminoglycosides and fluoroquinolones displayed a range of activities against the RGM isolates. Amikacin displayed the highest activity $(72 / 73,98.63 \%)$, while moxifloxacin displayed a range of activities (57/73, 78.08\%). Tigecycline (70/73, 95.89\%) had much higher activity against the isolates than minocycline $(30 / 73,41.10 \%)$ and doxycycline $(25 / 73,34.25 \%)$. Clarithromycin (48/73, 65.75\%), azithromycin (53/73, 72.60\%), and roxithromycin $(48 / 73,65.75 \%)$ showed various in vitro activities against the RGM isolates. Meropenem (52/73, $71.23 \%)$ exhibited good activity against the strains tested. Cefmetazole $(62 / 73,84.93 \%)$ was more active than cefoxitin (49/73, 67.12\%) and amoxicillin-clavulanic acid (11/73, $15.07 \%)$. In contrast, linezolid (71/73, 97.26\%) was highly active against the majority of the RGM isolates. Clofazimine $(47 / 73,64.38 \%)$ and sulfamethoxazole $(56 / 73,76.71 \%)$ inhibited the majority of isolates. Rifapentine $(5 / 73,6.85 \%)$ and rifabutin $(26 / 73,35.62 \%)$, which are rifamycin derivatives, were better than rifampicin. However, dapsone (5/73, 6.85\%), thioacetazone $(0 / 73,0 \%)$, and pasiniazid $(0 / 73,0 \%)$ displayed poor activities against the RGM isolates.

The MICs, $\mathrm{MIC}_{50}$, and $\mathrm{MIC}_{90}$ ranges for each antimicrobial agent tested against each RGM species are shown in Table 4. Based on the $\mathrm{MIC}_{90}$ values of the isolates, capreomycin was $8 \mu \mathrm{g} / \mathrm{mL}$ for $M$. abscessus and $2 \mu \mathrm{g} / \mathrm{mL}$ for $M$. fortuitum. The $\mathrm{MIC}_{50}$ value for levofloxacin was $8 \mu \mathrm{g} / \mathrm{mL}$ for $M$. abscessus and $2 \mu \mathrm{g} / \mathrm{mL}$ for $M$. fortuitum. Clofazimine had very high activity, with $\mathrm{MIC}_{90}$ values of $8 \mu \mathrm{g} / \mathrm{mL}$ for $M$. abscessus and $2 \mu \mathrm{g} / \mathrm{mL}$ for $M$. fortuitum. 
TABLE 2: MIC $(\mu \mathrm{g} / \mathrm{mL})$ results of the antimicrobial susceptibility tests for five rapidly growing reference mycobacteria.

\begin{tabular}{|c|c|c|c|c|c|c|c|c|c|c|c|c|c|c|c|c|}
\hline Sp. & RFP & INH & EMB & SM & $\mathrm{AM}$ & $\mathrm{KN}$ & CPM & TOB & OF & CIP & LEV & SPA & MXF & LNZ & CLO & SMZ \\
\hline M. abscessus & 64 & $>256$ & 32 & 16 & 1 & 2 & 0.5 & 8 & 4 & 2 & $\underline{1}$ & 2 & 1 & 4 & 0.06 & 32 \\
\hline M. chelonae & $>256$ & $>256$ & 128 & 32 & 4 & 16 & 16 & 2 & 32 & 4 & 16 & 16 & $T$ & 8 & 0.25 & 64 \\
\hline M. fortuitum & 128 & 64 & 256 & 32 & 0.25 & 4 & $<0.03$ & 16 & 0.13 & 0.03 & 0.03 & $<0.03$ & $<0.03$ & 8 & 0.03 & 2 \\
\hline M. septicum & 16 & 256 & 4 & 16 & 0.13 & 0.5 & $<0.03$ & 1 & 0.25 & 0.06 & 0.13 & 0.03 & 0.06 & 1 & 0.13 & 1 \\
\hline M. neoaurum & 1 & $>256$ & 8 & 0.5 & 0.5 & 0.06 & $<0.03$ & 0.25 & 0.06 & 0.06 & 0.03 & $<0.03$ & $<0.03$ & 0.5 & 0.03 & 0.5 \\
\hline Sp. & MIN & DOX & TIG & CLR & AZM & ROX & AMC & FOX & $\mathrm{CMZ}$ & MEM & RFT & RFB & DAP & THI & \multicolumn{2}{|c|}{ PASI } \\
\hline M. abscessus & 8 & 32 & 4 & $<0.03$ & $<0.03$ & 0.03 & $>256$ & 32 & 32 & 64 & 0.13 & 32 & 64 & 16 & \multicolumn{2}{|c|}{32} \\
\hline M. chelonae & 64 & $>32$ & 1 & 0.06 & 0.5 & 2 & $>256$ & 128 & $<0.25$ & $>256$ & 0.13 & 32 & 32 & $>256$ & \multicolumn{2}{|c|}{$>256$} \\
\hline M. fortuitum & 8 & 0.03 & 0.5 & 1 & 2 & 4 & $>256$ & 32 & 4 & 4 & 1 & 16 & 8 & 256 & \multicolumn{2}{|c|}{2} \\
\hline M. septicum & 32 & 8 & 1 & 1 & 16 & 4 & $>256$ & 128 & 1 & 16 & 0.13 & $<0.03$ & 32 & 256 & \multicolumn{2}{|c|}{4} \\
\hline M. neoaurum & 0.03 & 0.06 & 0.03 & 1 & 1 & 2 & 32 & 8 & 1 & 0.5 & 0.06 & 0.13 & 4 & $>256$ & \multicolumn{2}{|c|}{64} \\
\hline
\end{tabular}

Note 1: M. abscessus: ATCC19977; M. chelonae: ATCC35752; M. fortuitum: DSM44220; M. neoaurum: ATCC25795; and M. septicum: DSM44393.

Note 2: INH: isoniazid; RFP: rifampicin; EMB: ethambutol; SM: streptomycin; AM: amikacin; KN: kanamycin; CPM: capreomycin; TOB: tobramycin; OF: ofloxacin; CIP: ciprofloxacin; LEV: levofloxacin; SPA: sparfloxacin; MXF: moxifloxacin; LNZ: linezolid; CLO: clofazimine; SMZ: sulfamethoxazole; CLR: clarithromycin; AZM: azithromycin; ROX: roxithromycin; MIN: minocycline; DOX: doxycycline; TIG: tigecycline; AMC: Amoxicillin-clavulanic Acid; FOX: cefoxitin; CMZ: cefmetazole; MEM: meropenem; RPT: rifapentine; RBT: rifabutin; DAP: dapsone; THI: thioacetazone; PASI: pasiniazid.

Note 3: Bold, italic values indicate drug susceptibility. Values shown in bold indicate moderate drug susceptibility.

The $\mathrm{MIC}_{50}$ and $\mathrm{MIC}_{90}$ values of doxycycline were $16 \mu \mathrm{g} / \mathrm{mL}$ and $32 \mu \mathrm{g} / \mathrm{mL}$, respectively, for M. abscessus, and $64 \mu \mathrm{g} / \mathrm{mL}$ and $256 \mu \mathrm{g} / \mathrm{mL}$, respectively, for M. fortuitum. Azithromycin had better activity against $M$. abscessus than $M$. fortuitum, with $\mathrm{MIC}_{50}$ values of $0.5 \mu \mathrm{g} / \mathrm{mL}$ and $2 \mu \mathrm{g} / \mathrm{mL}$, respectively.

\section{Discussion}

With the development of improved microbiological and laboratory techniques, more RGM have been identified [20]. Effective treatment of RGM-related diseases is challenging to physicians because it is not obvious which drugs should be selected. In this study, the susceptibilities of 73 clinical RGM isolates and their corresponding reference RGM strains were examined for 31 antimicrobial agents using CAMH broth microdilution methodology.

Some studies have shown that $M$. abscessus, $M$. fortuitum, and $M$. chelonae are important human pathogens among RGM isolates [21-24]. Here, we showed that M. abscessus $(75.34 \%)$ is the predominant RGM species in the 73 clinical isolates, followed by $M$. fortuitum (15.07\%). Both organisms were susceptible to amikacin, linezolid, tigecycline, cefmetazole, capreomycin, moxifloxacin, macrolides, and carbapenems, but were highly resistant to the firstline antituberculous drugs, dapsone, thioacetazone, and pasiniazid. The percentage of resistance to numerous drugs was higher in $M$. abscessus than in M. fortuitum, except for moxifloxacin, minocycline, doxycycline, roxithromycin, cefmetazole, and rifabutin. In a recent report, amikacin and clarithromycin were the optimal choices against infection with $M$. abscessus [25]. Additionally, quinolones and trimethoprim-sulfamethoxazole were effective against $M$. fortuitum [25]. In the present study, Amikacin was the most active drug against $M$. abscessus. Furthermore, we found that amikacin, capreomycin, and linezolid had the highest antibacterial activities against $M$. fortuitum.
Previous studies have reported that numerous RGM strains were highly resistant to the first-line antituberculous agents [26, 27]. Our data confirms this finding. Elsewhere, researchers have shown that dapsone had little activity against RGM isolates [18]. Thioacetazone is used mainly as an antituberculous agent but has variable activity, and the drug was formerly used in conjunction with isoniazid [19]. RGM strains have been shown to be highly resistant to pasiniazid [28]. Our data shows that dapsone had little activity against M. abscessus and M. fortuitum isolates, while thioacetazone and pasiniazid had no activity against any of the RGM organisms.

Aminoglycosides and quinolones, which are second-line antituberculous drugs, have good activities against RGM strains [28-30]. In our study, amikacin was found to have potential to be effective for treatment of RGM diseases and showed higher activity than the other aminoglycoside antibiotics we tested. However, a higher percentage of $M$. chelonae isolates were sensitive to tobramycin than $M$. abscessus, although the sample size of the latter was smaller. The third generation fluoroquinolone drugs levofloxacin and sparfloxacin displayed higher activities than ofloxacin. Moxifloxacin, a fourth generation fluoroquinolone, displayed higher activity than the third generation ones [30]. Quinolones exhibited better activity against $M$. fortuitum than $M$. abscessus, especially levofloxacin, against which $M$. fortuitum was more susceptible than M. abscessus.

Minocycline, doxycycline, and tigecycline represent the newest tetracycline derivatives [31]. A recent study [26] showed that NTM displayed $\sim 50 \%$ susceptibility to doxycycline and minocycline, but in our research susceptibility to these two drugs was more than 20\% (Table 3). This finding may reflect the small sample number of $M$. fortuitum in this study. Tigecycline displayed activity against RGM organisms [26]. We found that tigecycline had more activity than minocycline and doxycycline, with lower $\mathrm{MIC}_{50}$ and $\mathrm{MIC}_{90}$ values. 
TABLE 3: In vitro drug susceptibility percentage per species for rapidly growing mycobacteria isolates.

\begin{tabular}{|c|c|c|c|c|c|c|}
\hline \multirow[b]{2}{*}{ Drugs } & \multicolumn{5}{|c|}{ Species } & \multirow{2}{*}{$\begin{array}{c}\text { Total }(n=73) \\
(\%)\end{array}$} \\
\hline & $\begin{array}{l}\text { M. abscessus } \\
(n=55)(\%)\end{array}$ & $\begin{array}{l}\text { M. fortuitum } \\
(n=11)(\%)\end{array}$ & $\begin{array}{c}\text { M. chelonae } \\
(n=3)(\%)\end{array}$ & $\begin{array}{c}\text { M. septicum } \\
(n=2)(\%)\end{array}$ & $\begin{array}{c}\text { M. neoaurum } \\
(n=2)(\%)\end{array}$ & \\
\hline INH & $0(0)$ & $0(0)$ & $0(0)$ & $0(0)$ & $0(0)$ & $0(0)$ \\
\hline RFP & $3(5.46)$ & $0(0)$ & $0(0)$ & $0(0)$ & $0(0)$ & $3(4.11)$ \\
\hline EMB & $2(3.64)$ & $0(0)$ & $0(0)$ & $0(0)$ & $0(0)$ & $2(2.74)$ \\
\hline SM & $3(5.46)$ & $0(0)$ & $0(0)$ & $0(0)$ & $1(50.00)$ & $4(5.48)$ \\
\hline $\mathrm{AM}$ & $55(100.00)$ & $11(100.00)$ & $3(100.00)$ & $2(100.00)$ & $1(50.00)$ & $72(98.63)$ \\
\hline $\mathrm{KN}$ & 27 (49.09) & $5(45.45)$ & $1(33.33)$ & $1(50.00)$ & $0(0)$ & $34(46.58)$ \\
\hline CPM & $41(74.55)$ & $11(100.00)$ & $1(33.33)$ & $2(100.00)$ & $0(0)$ & $55(75.34)$ \\
\hline TOB & $25(45.45)$ & $6(54.55)$ & $3(100.00)$ & $1(50.00)$ & $0(0)$ & $35(47.94)$ \\
\hline OF & $9(16.36)$ & $5(45.45)$ & $0(0)$ & $2(100.00)$ & $2(100.00)$ & $18(24.66)$ \\
\hline CIP & $24(43.64)$ & $5(45.45)$ & $1(33.33)$ & $2(100.00)$ & $1(50.00)$ & $33(45.21)$ \\
\hline LEV & $26(47.27)$ & $8(72.73)$ & $2(66.67)$ & $2(100.00)$ & $1(50.00)$ & $39(53.42)$ \\
\hline SPA & $23(41.82)$ & $6(54.55)$ & $3(100.00)$ & $2(100.00)$ & $1(50.00)$ & $35(47.94)$ \\
\hline MXF & $43(78.18)$ & $8(72.73)$ & $3(100.00)$ & $2(100.00)$ & $1(50.00)$ & $57(78.08)$ \\
\hline LNZ & $53(96.36)$ & $11(100.00)$ & $3(100.00)$ & $2(100.00)$ & $2(100.00)$ & $71(97.26)$ \\
\hline CLO & $35(63.64)$ & $8(72.73)$ & $1(33.33)$ & $2(100.00)$ & $1(50.00)$ & $47(64.38)$ \\
\hline SMZ & $41(74.55)$ & $10(90.91)$ & $2(66.67)$ & $2(100.00)$ & $1(50.00)$ & $56(76.71)$ \\
\hline MIN & $24(43.64)$ & $3(27.27)$ & $2(66.67)$ & $1(50.00)$ & $0(0)$ & $30(41.10)$ \\
\hline DOX & $19(34.55)$ & $3(27.27)$ & $2(66.67)$ & $0(0)$ & $1(50.00)$ & $25(34.25)$ \\
\hline TIG & $53(96.36)$ & $10(90.91)$ & $3(100.00)$ & $2(100.00)$ & $2(100.00)$ & 70 (95.89) \\
\hline CLR & $37(67.27)$ & $7(63.64)$ & $2(66.67)$ & $1(50.00)$ & $1(50.00)$ & $48(65.75)$ \\
\hline AZM & $43(78.18)$ & $6(54.55)$ & $2(66.67)$ & $0(0)$ & $2(100.00)$ & $53(72.60)$ \\
\hline ROX & $38(69.09)$ & $7(63.64)$ & $2(66.67)$ & $0(0)$ & $1(50.00)$ & $48(65.75)$ \\
\hline AMC & $10(18.18)$ & $0(0)$ & $1(33.33)$ & $0(0)$ & $0(0)$ & $11(15.07)$ \\
\hline FOX & $38(69.09)$ & $9(81.82)$ & $1(33.33)$ & $0(0)$ & $1(50.00)$ & $49(67.12)$ \\
\hline CMZ & $48(87.27)$ & $9(81.82)$ & $2(66.67)$ & $1(50.00)$ & $2(100.00)$ & $62(84.93)$ \\
\hline MEM & 39 (70.91) & $8(72.73)$ & $1(33.33)$ & $2(100.00)$ & $2(100.00)$ & $52(71.23)$ \\
\hline RPT & $4(7.27)$ & $0(0)$ & $1(33.33)$ & $0(0)$ & $0(0)$ & $5(6.85)$ \\
\hline RBT & $22(40.00)$ & 1 (9.09) & $2(66.67)$ & $0(0)$ & $1(50.00)$ & $26(35.62)$ \\
\hline DAP & $4(7.27)$ & $1(9.09)$ & $0(0)$ & $0(0)$ & $0(0)$ & $5(6.85)$ \\
\hline THI & $0(0)$ & $0(0)$ & $0(0)$ & $0(0)$ & $0(0)$ & $0(0)$ \\
\hline PASI & $0(0)$ & $0(0)$ & $0(0)$ & $0(0)$ & $0(0)$ & $0(0)$ \\
\hline
\end{tabular}

Note: $n$ : number of strains tested.

RGM strains have been shown to be susceptible to the newer generation of macrolide antibiotics (i.e., clarithromycin, azithromycin, and roxithromycin) $[25,26,30]$. This drug class is a good alternative for treating RGM species because of its high activity and oral formulations. Clinical experience shows that azithromycin toxicity is dose dependent and most adult patients with M. avium complex (MAC) lung disease do not tolerate azithromycin doses greater than $300 \mathrm{mg} /$ day because of frequent of adverse events, including gastrointestinal symptoms (primarily diarrhea) and reversible hearing impairment [26]. In our research, the isolates were less susceptible to clarithromycin than to azithromycin, making the latter more applicable in the future. Some reports suggest that clarithromycin resistance can be induced in M. abscessus and M. fortuitum, and the resistance is associated with erm (41) and erm (39) genes, respectively $[26,32]$. The M. abscessus complex is subclassified into three closely related subspecies (M. abscessus, M. massiliense, and $M$. bolletii) [33]. Historically, the $M$. fortuitum group has included three species: M. fortuitum, M. peregrinum, and an unnamed third biovariant complex [34]. The different subspecies potentially exhibit different drug susceptibilities. Macrolide resistance in M. abscessus and M. fortuitum will be the subject of our future research.

Cefoxitin and cefmetazole are second and third generation cephalosporin antibiotics and in previous studies cefoxitin was frequently found to have good mycobacterial activity $[25,26,28,30]$. The isolates were more susceptible to cefmetazole than cefoxitin, so cefmetazole can be used where cefoxitin is ineffective. In addition, the antibacterial 
TABLE 4: The MIC range, $\mathrm{MIC}_{50}$, and $\mathrm{MIC}_{90}(\mu \mathrm{g} / \mathrm{mL})$ per species of rapidly growing mycobacterial isolates for all the antibacterial agents tested.

\begin{tabular}{|c|c|c|c|c|c|c|c|c|c|}
\hline \multirow{2}{*}{ Drugs } & \multicolumn{3}{|c|}{ M. abscessus $(n=55)$} & \multicolumn{3}{|c|}{ M. fortuitum $(n=11)$} & \multicolumn{3}{|c|}{ Other species $(n=7)$} \\
\hline & MIC range & $\mathrm{MIC}_{50}$ & $\mathrm{MIC}_{90}$ & MIC range & $\mathrm{MIC}_{50}$ & $\mathrm{MIC}_{90}$ & MIC range & $\mathrm{MIC}_{50}$ & $\mathrm{MIC}_{90}$ \\
\hline RFP & $0.5->256$ & 16 & 128 & $1->256$ & 128 & $>256$ & $2-8$ & 2 & 8 \\
\hline INH & $2->256$ & $>256$ & $>256$ & $2->256$ & $>256$ & $>256$ & $16->256$ & $>256$ & $>256$ \\
\hline EMB & $1->256$ & $>256$ & $>256$ & $32->256$ & 256 & $>256$ & $128->256$ & $>256$ & $>256$ \\
\hline SM & $<0.25->256$ & 32 & 256 & $8->256$ & 32 & 128 & $4-64$ & 32 & 64 \\
\hline $\mathrm{AM}$ & $0.06-16$ & 4 & 8 & $0.06-16$ & 2 & 4 & $0.25->32$ & 4 & 8 \\
\hline $\mathrm{KN}$ & $0.25->32$ & 4 & 32 & $0.5->32$ & 8 & 16 & $2->32$ & 16 & $>32$ \\
\hline $\mathrm{CPM}$ & $0.03-16$ & 1 & 8 & $0.03-2$ & 0.5 & 2 & $0.03-32$ & 4 & 16 \\
\hline TOB & $<0.25-64$ & 8 & 32 & $0.5-32$ & 4 & 32 & $0.5-32$ & 4 & 16 \\
\hline OF & $0.06-32$ & 8 & 16 & $0.06-32$ & 4 & 16 & $0.03-8$ & 0.5 & 8 \\
\hline CIP & $0.03-32$ & 4 & 4 & $0.06-8$ & 4 & 8 & $0.13-8$ & 2 & 4 \\
\hline LEV & $0.03-32$ & 8 & 8 & $0.06-8$ & 2 & 8 & $0.13-16$ & 4 & 8 \\
\hline SPA & $0.03-32$ & 4 & 16 & $0.03-16$ & 2 & 16 & $0.06-16$ & 0.25 & 2 \\
\hline MXF & $0.03-16$ & 2 & 4 & $0.03-8$ & 1 & 4 & $0.06-8$ & 0.5 & 2 \\
\hline LNZ & $0.5->64$ & 4 & 16 & $0.5-16$ & 4 & 16 & $0.25-16$ & 4 & 8 \\
\hline CLO & $0.06->32$ & 0.25 & 8 & $0.03-16$ & 0.25 & 2 & $0.06->32$ & 0.5 & 4 \\
\hline SMZ & $0.5->256$ & 32 & $>256$ & $4-128$ & 16 & 64 & $4->256$ & 16 & 256 \\
\hline MIN & $<0.25->256$ & 16 & 32 & $1-256$ & 16 & 64 & $2-32$ & 16 & 32 \\
\hline DOX & $<0.25->256$ & 16 & 32 & $<0.25-256$ & 64 & 256 & $1-64$ & 8 & 32 \\
\hline TIG & $<0.25-8$ & 2 & 2 & $<0.25-8$ & 4 & 4 & $<0.25-1$ & 0.5 & 1 \\
\hline CLR & $0.03->32$ & 0.13 & $>32$ & $0.06->32$ & 0.25 & 16 & $0.03->32$ & 1 & 8 \\
\hline $\mathrm{AZM}$ & $0.03->32$ & 0.5 & $>32$ & $0.13->32$ & 2 & $>32$ & $0.13->32$ & 1 & 32 \\
\hline ROX & $0.03->32$ & 0.25 & $>32$ & $0.13-32$ & 1 & 32 & $0.03-16$ & 8 & 16 \\
\hline AMC & $0.5->256$ & $>256$ & $>256$ & $256->256$ & $>256$ & $>256$ & $1->256$ & $>256$ & $>256$ \\
\hline FOX & $4->256$ & 64 & 256 & $16->256$ & 32 & 128 & $32->256$ & 128 & 256 \\
\hline CMZ & $1-128$ & 16 & 64 & $8-64$ & 16 & 64 & $8->256$ & 32 & 64 \\
\hline MEM & $<0.25->256$ & 8 & 128 & $<0.25->256$ & 8 & 256 & $0.5-32$ & 4 & 32 \\
\hline RPT & $0.03->32$ & 8 & 32 & $1->32$ & 8 & $>32$ & $0.06-32$ & 2 & 16 \\
\hline RBT & $0.03->32$ & 1 & 16 & $0.5->32$ & 2 & 16 & $0.03-4$ & 1 & 4 \\
\hline DAP & $1->256$ & 16 & 128 & $2->256$ & 64 & 128 & $4-256$ & 16 & 64 \\
\hline THI & $8->256$ & $>256$ & $>256$ & $128->256$ & $>256$ & $>256$ & $128->256$ & $>256$ & $>256$ \\
\hline PASI & $2->256$ & 256 & $>256$ & $8->256$ & $>256$ & $>256$ & $16->256$ & $>256$ & $>256$ \\
\hline
\end{tabular}

Note: $n$ : number of strains tested.

mechanism for meropenem, a carbapenem antibiotic, occurs via inhibition of bacterial cell wall synthesis [35]. Carbapenems include imipenem, meropenem, and ertapenem, among others. In previous studies, imipenem was widely used in experiments $[25,26,30]$. Here, among the RGM strains, meropenem was found to have good activity and a lower MIC value than cefoxitin. In clinical work, rifabutin has been used mainly to target SGM, and toxicity to this drug was dose related. Clarithromycin has been shown to increase rifabutin serum levels and this effect was likely to be related to the hepatic metabolism of rifabutin [26]. Our data indicates that rifabutin and rifapentine can be used to treat rifampicinresistant strains; the RGM isolates have better susceptibility to rifabutin than rifapentine, but rifapentine was more active against $M$. chelonae, M. abscessus, and M. fortuitum.
Otherwise, the rifabutin dose should be reduced when used in combination with clarithromycin to treat infections with RGM strains.

Several studies have shown that linezolid and clofazimine have potent activities against NTM [25, 26, 29, 30, 36]. In our experiments, linezolid had $>95 \%$ activity against the strains tested and clofazimine $>60 \%$ susceptibility for the RGM isolates (with the exception of $M$. chelonae). The small $M$. chelonae sample size probably affected this result. In the future, a larger sample size should be used to determine the MICs of the 31 drugs used in this study.

The RGM isolates used in our research were from four different Chinese provinces. Most of them $(86.30 \%)$ were from Fujian Province, making it important to obtain more samples from different geographical areas in the future. 
The data presented here suggest that tigecycline or linezolid combined with clofazimine or cefmetazole should be the most efficacious drug combination for treating M. abscessus infections. For $M$. fortuitum, capreomycin, sulfamethoxazole, tigecycline, clofazimine, and cefmetazole in combination may be a good choice.

\section{Conclusions}

In summary, our data provide useful information on antibiotics that are effective against RGM and this information may help to identify suitable therapy for patients infected with such organisms. Future studies should address whether combining two or more antimicrobial agents for treatment of RGM infections is better than treatment with a single drug alone.

\section{Conflict of Interests}

The authors have declared that no conflict of interests exists.

\section{Acknowledgments}

This work was financially supported by the 2013ZX10004101, and 2013ZX10003002-001 project of the National Key Programme of Mega Infectious Diseases, the Key Project (2014SKLID104) of the State Key Laboratory for Infectious Disease Prevention and Control, and a Science and Technology Innovation Team support project (CX201412) from Changzhi Medical College. The authors thank the staff at the respective institutes and hospitals in Mingxiang Huang (Fujian). Yunhong Tan (Hunan) and Chongxiang Tong (Gansu) contributed to this study by collecting clinical isolates and patient information.

\section{References}

[1] J. E. Stout, L. B. Gadkowski, S. Rath, J. A. Alspaugh, M. B. Miller, and G. M. Cox, "Pedicure-associated rapidly growing mycobacterial infection: an endemic disease," Clinical Infectious Diseases, vol. 53, no. 8, pp. 787-792, 2011.

[2] L. Wang, H. Zhang, Y. Ruan et al., "Tuberculosis prevalence in China, 1990-2010; a longitudinal analysis of national survey data," The Lancet, vol. 383, no. 9934, pp. 2057-2064, 2014.

[3] G. El Helou, G. M. Viola, R. Hachem, X. Y. Han, and I. I. Raad, "Rapidly growing mycobacterial bloodstream infections," The Lancet Infectious Diseases, vol. 13, no. 2, pp. 166-174, 2013.

[4] A. C. Helguera-Repetto, R. Chacon-Salinas, J. F. Cerna-Cortes et al., "Differential macrophage response to slow- and fastgrowing pathogenic mycobacteria," BioMed Research International, vol. 2014, Article ID 916521, 10 pages, 2014.

[5] W. J. Kheir, H. Sheheitli, M. Abdul Fattah, and R. N. Hamam, "Nontuberculous mycobacterial ocular infections: a systematic review of the literature," BioMed Research International, vol. 2015, Article ID 164989, 17 pages, 2015.

[6] R. E. Colombo and K. N. Olivier, "Diagnosis and treatment of infections caused by rapidly growing mycobacteria," Seminars in Respiratory and Critical Care Medicine, vol. 29, no. 5, pp. 577$588,2008$.
[7] C. Bicmen, M. Coskun, A. T. Gunduz, G. Senol, A. Kadri Cirak, and G. Tibet, "Nontuberculous mycobacteria isolated from pulmonary specimens between 2004 and 2009: causative agent or not?" New Microbiologica, vol. 33, no. 4, pp. 399-403, 2010.

[8] Clinical and Laboratory Standards Institute, Susceptibility Testing of Mycobacteria, Nocardiae, and Other Aerobic Actinomycetes; Approved Standard-Second Edition, CLSI Document, M24-A2, 2011.

[9] J.-U. Kim, C.-H. Cha, and H.-K. An, "Multiplex real-time PCR assay and melting curve analysis for identifying Mycobacterium tuberculosis complex and nontuberculous mycobacteria," Journal of Clinical Microbiology, vol. 50, no. 2, pp. 483-487, 2012.

[10] A. C. Pérez-Osorio, D. S. Boyle, Z. K. Ingham et al., "Rapid identification of mycobacteria and drug-resistant Mycobacterium tuberculosis by use of a single multiplex PCR and DNA sequencing," Journal of Clinical Microbiology, vol. 50, no. 2, pp. 326-336, 2012.

[11] World Health Organization, "Policy guidance on drugsusceptibility testing (DST) of second-line antituberculosis drugs," WHO Document, World Health Organization, 2008.

[12] E. Kłosińska-Szmurlo, F. A. Pluciński, M. Grudzień, K. Betlejewska-Kielak, J. Biernacka, and A. P. Mazurek, "Experimental and theoretical studies on the molecular properties of ciprofloxacin, norfloxacin, pefloxacin, sparfloxacin, and gatifloxacin in determining bioavailability," Journal of Biological Physics, vol. 40, no. 4, pp. 335-345, 2014.

[13] M. Gopal, N. Padayatchi, J. Z. Metcalfe, and M. R. O’Donnell, "Systematic review of clofazimine for the treatment of drugresistant tuberculosis," International Journal of Tuberculosis and Lung Disease, vol. 17, no. 8, pp. 1001-1007, 2013.

[14] Z. Samra, S. Rosenberg, and M. Dan, "Susceptibility of Ureaplasma urealyticum to tetracycline, doxycycline, erythromycin, roxithromycin, clarithromycin, azithromycin, levofloxacin and moxifloxacin," Journal of Chemotherapy, vol. 23, no. 2, pp. 77-79, 2011.

[15] J. Larruskain, P. Idigoras, J. M. Marimon, and E. Perez-Trallero, "Susceptibility of 186 Nocardia sp. isolates to 20 antimicrobial agents," Antimicrobial Agents and Chemotherapy, vol. 55, no. 6, pp. 2995-2998, 2011.

[16] Clinical and Laboratory Standards Institute, "Performance standards for antimicrobial susceptibility testing; twenty first informational supplement," CLSI Document M100-S21, Clinical and Laboratory Standards Institute, 2011.

[17] T. Staudinger, B. Redl, and B. J. Glasgow, "Antibacterial activity of rifamycins for $M$. smegmatis with comparison of oxidation and binding to tear lipocalin," Biochimica et Biophysica Acta, vol. 1844, no. 4, pp. 750-758, 2014.

[18] G.-H. Shen, B.-D. Wu, S.-T. Hu, C.-F. Lin, K.-M. Wu, and J.-H. Chen, "High efficacy of clofazimine and its synergistic effect with amikacin against rapidly growing mycobacteria," International Journal of Antimicrobial Agents, vol. 35, no. 4, pp. 400-404, 2010.

[19] G. D. Coxon, D. Craig, R. M. Corrales, E. Vialla, L. GannounZaki, and L. Kremer, "Synthesis, antitubercular activity and mechanism of resistance of highly effective thiacetazone analogues," PLoS ONE, vol. 8, no. 1, Article ID e53162, 2013.

[20] E. E. McGrath and P. B. Anderson, "The therapeutic approach to non-tuberculous mycobacterial infection of the lung," Pulmonary Pharmacology and Therapeutics, vol. 23, no. 5, pp. 389396, 2010. 
[21] Y. Shao, C. Chen, H. Song et al., "The he epidemiology and geographic distribution of nontuberculous mycobacteria clinical isolates from sputum samples in the eastern region of china," PLOS Neglected Tropical Diseases, vol. 9, no. 3, Article ID e0003623, 2015.

[22] A. A. Velayati, P. Farnia, M. Mozafari et al., "Molecular epidemiology of nontuberculous mycobacteria isolates from clinical and environmental sources of a metropolitan city," PLoS ONE, vol. 9, no. 12, Article ID el14428, 2014.

[23] T. Qvist, M. Gilljam, B. Jönsson et al., "Epidemiology of nontuberculous mycobacteria among patients with cystic fibrosis in Scandinavia," Journal of Cystic Fibrosis, vol. 14, no. 1, pp. 46-52, 2015.

[24] M. Panagiotou, A. I. Papaioannou, K. Kostikas et al., "The epidemiology of pulmonary nontuberculous mycobacteria: data from a general hospital in Athens, Greece, 2007-2013," Pulmonary Medicine, vol. 2014, Article ID 894976, 9 pages, 2014.

[25] S. S. Tang, D. C. Lye, R. Jureen et al., "Rapidly growing mycobacteria in Singapore, 2006-2011," Clinical Microbiology and Infection, vol. 21, no. 3, pp. 236-241, 2015.

[26] D. E. Griffith, T. Aksamit, B. A. Brown-Elliott et al., "An official ATS/IDSA statement: diagnosis, treatment, and prevention of nontuberculous mycobacterial diseases," American Journal of Respiratory and Critical Care Medicine, vol. 175, no. 4, pp. 367416, 2007.

[27] A. H. Shahraki, P. Heidarieh, S. Z. Bostanabad et al., "'Multidrug-resistant tuberculosis' may be nontuberculous mycobacteria," European Journal of Internal Medicine, vol. 26, no. 4, pp. 279-284, 2015.

[28] R. Gayathri, K. L. Therese, P. Deepa, S. Mangai, and H. N. Madhavan, "Antibiotic susceptibility pattern of rapidly growing mycobacteria," Journal of Postgraduate Medicine, vol. 56, no. 2, pp. 76-78, 2010.

[29] G. Li, L.-L. Lian, L. Wan et al., "Antimicrobial susceptibility of standard strains of nontuberculous mycobacteria by microplate Alamar Blue assay," PLoS ONE, vol. 8, no. 12, Article ID e84065, 2013.

[30] S. Park, S. Kim, E. M. Park et al., "In vitro antimicrobial susceptibility of Mycobacterium abscessus in Korea," Journal of Korean Medical Science, vol. 23, no. 1, pp. 49-52, 2008.

[31] F. Nguyen, A. L. Starosta, S. Arenz, D. Sohmen, A. Dönhöfer, and D. N. Wilson, "Tetracycline antibiotics and resistance mechanisms," Biological Chemistry, vol. 395, no. 5, pp. 559-575, 2014.

[32] E. Chudáčková, T. Bergerová, K. Fajfrlík et al., "Carbapenemnonsusceptible strains of Klebsiella pneumoniae producing SHV-5 and/or DHA-1 $\beta$-lactamases in a Czech hospital," FEMS Microbiology Letters, vol. 309, no. 1, pp. 62-70, 2010.

[33] S. T. Howard, "Recent progress towards understanding genetic variation in the Mycobacterium abscessus complex," Tuberculosis, vol. 93, supplement 1, pp. S15-S20, 2013.

[34] R. J. Wallace Jr., G. Dukart, B. A. Brown-Elliott, D. E. Griffith, E. G. Scerpella, and B. Marshall, "Clinical experience in 52 patients with tigecycline-containing regimens for salvage treatment of Mycobacterium abscessus and Mycobacterium chelonae infections," Journal of Antimicrobial Chemotherapy, vol. 69, no. 7, pp. 1945-1953, 2014.

[35] Y.-K. Tsai, C.-H. Liou, C.-P. Fung, J.-C. Lin, and L. K. Siu, "Single or in combination antimicrobial resistance mechanisms of Klebsiella pneumoniae contribute to varied susceptibility to different carbapenems," PLoS ONE, vol. 8, no. 11, Article ID e79640, 2013.
[36] J. van Ingen, S. E. Totten, N. K. Helstrom, L. B. Heifets, M. J. Boeree, and C. L. Daley, "In vitro synergy between clofazimine and amikacin in treatment of nontuberculous mycobacterial disease," Antimicrobial Agents and Chemotherapy, vol. 56, no. 12, pp. 6324-6327, 2012. 

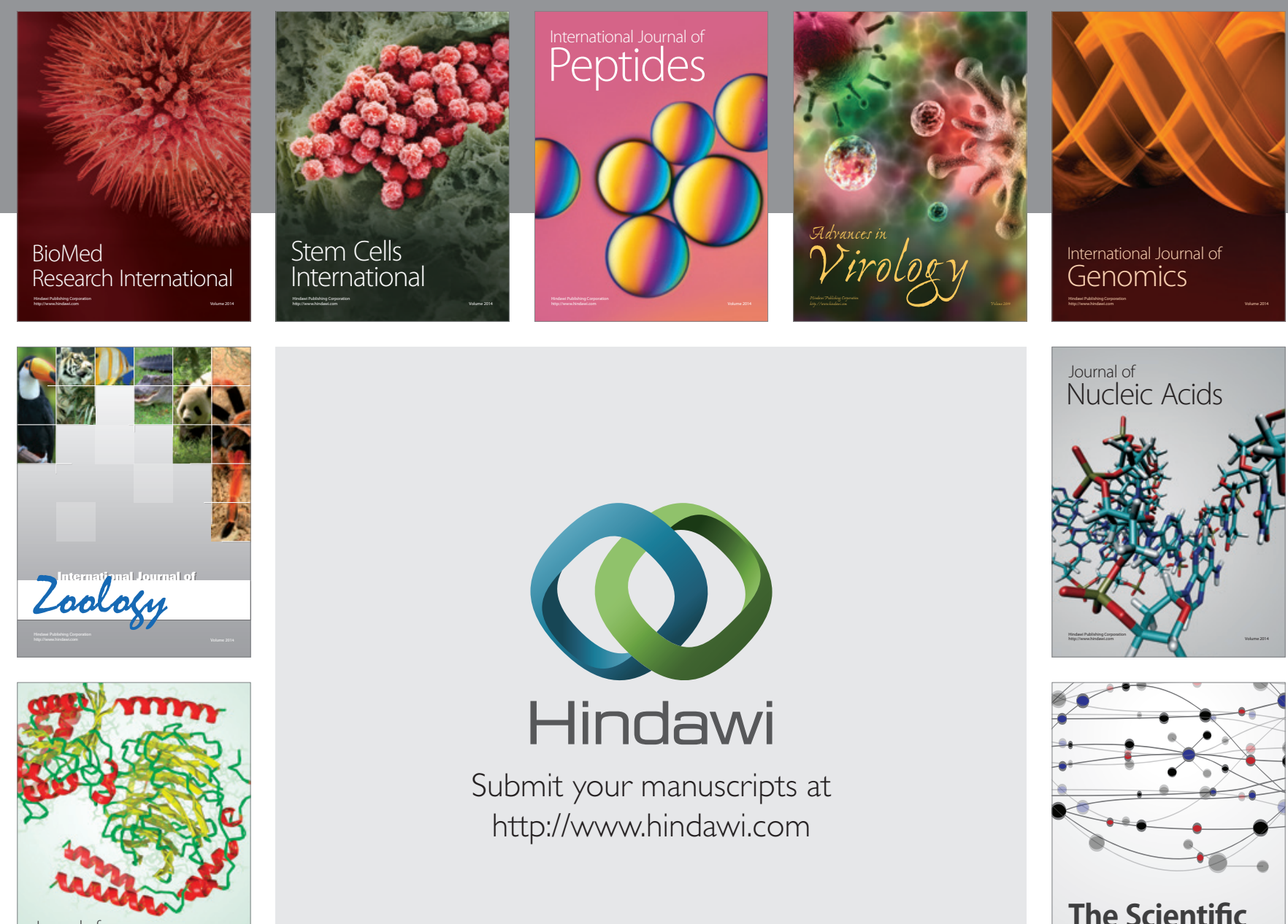

Submit your manuscripts at

http://www.hindawi.com

Journal of
Signal Transduction
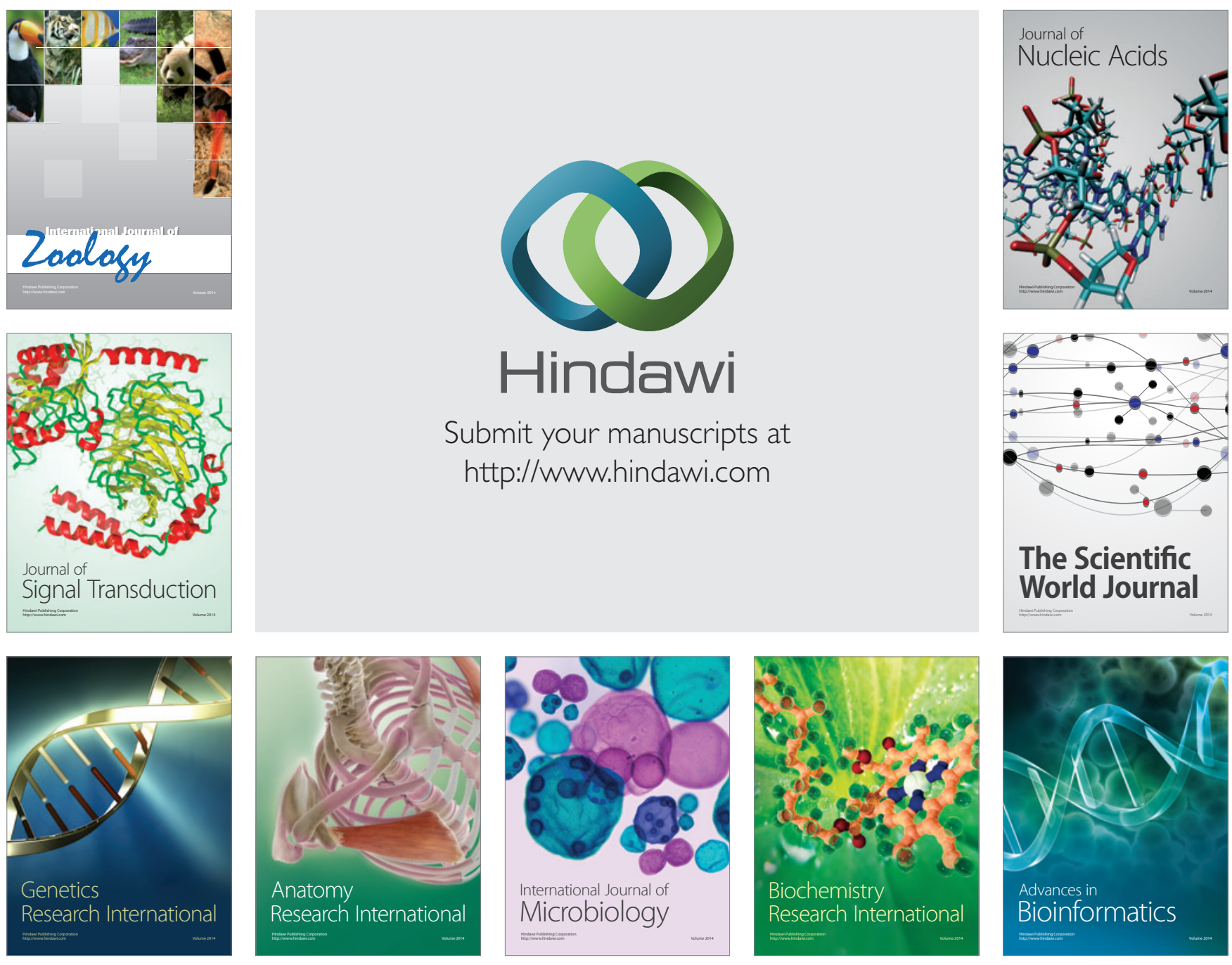

The Scientific World Journal
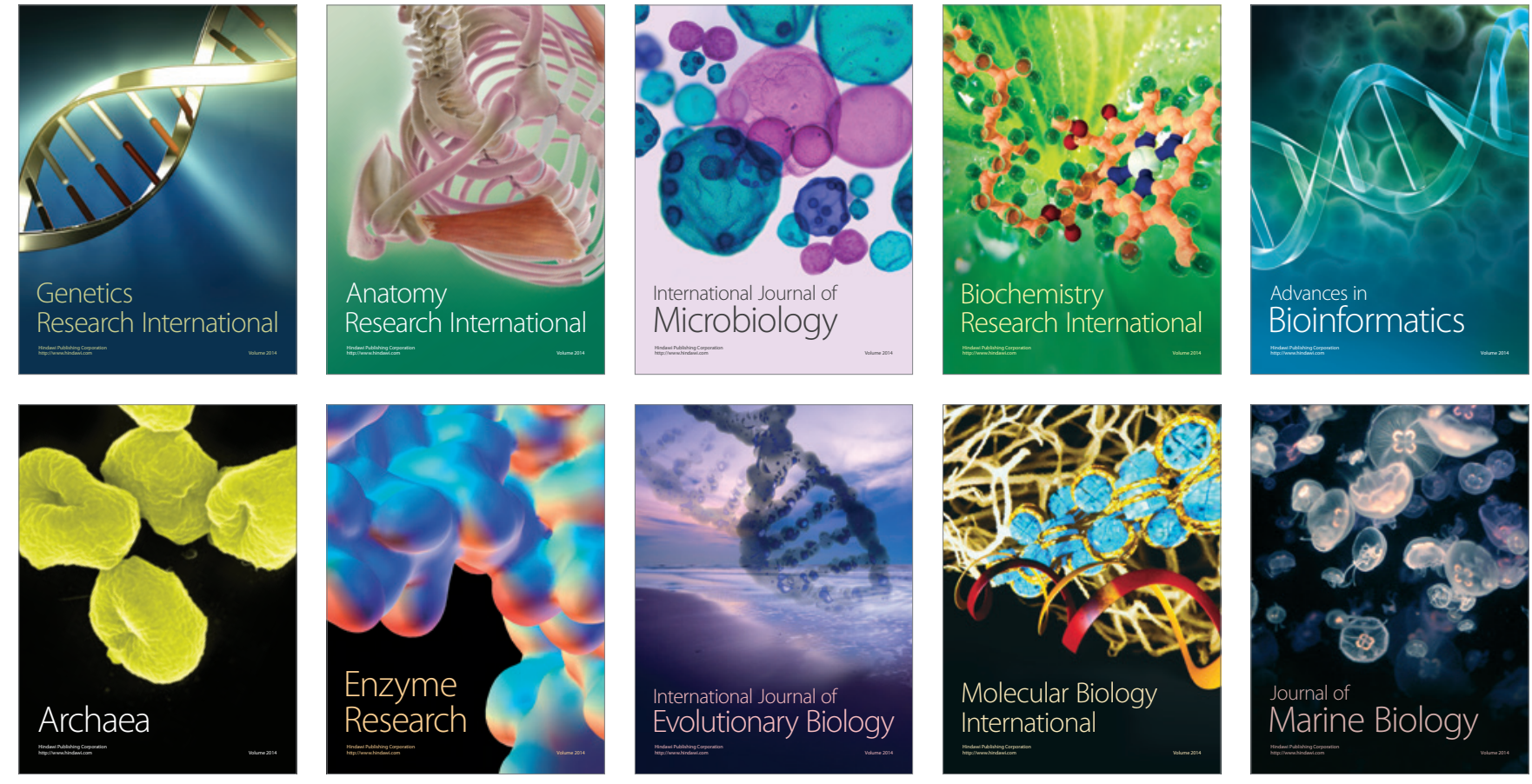